\title{
Daily functioning and self-management in patients with chronic low back pain after an intensive cognitive behavioral programme for pain management
}

\author{
Miranda L. van Hooff • Johannes D. van der Merwe • \\ John O'Dowd · Paul W. Pavlov • Maarten Spruit • \\ Marinus de Kleuver $\cdot$ Jacques van Limbeek
}

Received: 1 October 2009/Revised: 23 February 2010/Accepted: 2 May 2010/Published online: 27 May 2010

(C) The Author(s) 2010. This article is published with open access at Springerlink.com

\begin{abstract}
Chronic low back pain (CLBP) is associated with persistent or recurrent disability which results in high costs for society. Cognitive behavioral treatments produce clinically relevant benefits for patients with CLBP. Nevertheless, no clear evidence for the most appropriate intervention is yet available. The purpose of this study is to evaluate the mid-term effects of treatment in a cohort of patients with CLBP participating in an intensive pain management programme. The programme provided by RealHealth-Netherlands is based on cognitive behavioral principles and executed in collaboration with orthopedic surgeons. Main outcome parameters were daily functioning (Roland and Morris Disability Questionnaire and Oswestry Disability Questionnaire), self-efficacy (Pain Self-Efficacy Questionnaire) and quality of life (Short Form 36 Physical Component Score). All parameters were measured at baseline, last day of residential programme and at 1 and 12 months follow-up. Repeated measures analysis was applied to examine changes over time. Clinical relevance
\end{abstract}

M. L. van Hooff $(\bowtie) \cdot$ J. van Limbeek

Department Research Development and Education,

Sint Maartenskliniek, P.O. Box 9011, 6500 GM Nijmegen,

The Netherlands

e-mail: m.vanhooff@maartenskliniek.nl

J. D. van der Merwe · J. O'Dowd

The RealHealth Institute, 23-31 Beavor Lane,

London W6 9AR, UK

P. W. Pavlov · M. de Kleuver

Department of Orthopedics, Sint Maartenskliniek,

P.O. Box 9011, 6500 GM Nijmegen, The Netherlands

M. Spruit

Dubai Bone and Joint Centre LLC, Dubai Healthcare City,

Dubai, United Arab Emirates was examined using minimal clinical important differences (MCID) estimates for main outcomes. To compare results with literature effect sizes (Cohen's $d$ ) and Standardized Morbidity Ratios (SMR) were determined. 107 patients with CLBP participated in this programme. Mean scores on outcome measures showed a similar pattern: improvement after residential programme and maintenance of results over time. Effect sizes were 0.9 for functioning, 0.8 for self-efficacy and 1.3 for physical functioning related quality of life. Clinical relevancy: 79\% reached MCID on functioning, $53 \%$ on self-efficacy and $80 \%$ on quality of life. Study results on functioning were found to be $36 \%$ better and $2 \%$ worse when related to previous research on, respectively, rehabilitation programmes and spinal surgery for similar conditions (SMR 136 and 98\%, respectively). The participants of this evidence-based programme learned to manage CLBP, improved in daily functioning and quality of life. The study results are meaningful and comparable with results of spinal surgery and even better than results from less intensive rehabilitation programmes.

Keywords Chronic low back pain - Disability . Self-management $\cdot$ Cognitive behavioral programme . Cohort study $\cdot$ Clinical relevance

\section{Introduction}

Chronic low back pain (CLBP) is a major health and economic problem in western industrialized countries and it is associated with persistent or recurrent disability, resulting in high costs for society [1-3]. In the Netherlands in 2003 , the 1 year prevalence in the general population was estimated to be $44 \%$, and caused $24.4 \%$ of all sick leave in the employed population [4]. 
Annually, 22,000 new orthopedic patients visit the orthopedic surgery department at the Sint Maartenskliniek Nijmegen, in The Netherlands. Around 27\% $(6,000)$ are patients with low back pain complaints. Ten percent have an indication for surgery and another $10 \%$ for invasive pain treatment (epidural block, nerve root block, or spinal cord stimulation). Therefore, approximately $80 \%(4,500)$ of the CLBP sufferers do not need invasive treatment.

In literature there is no clear evidence that primary spinal fusion in patients with CLBP was any more beneficial than intensive rehabilitation [5, 6]. Fairbank et al. [5] came to this conclusion after they performed the MRC spine stabilization trial in which 349 patients were randomized to either a surgical stabilization (spinal fusion) or an intensive outpatient rehabilitation group (approximately $75 \mathrm{~h}$ of exercises, supported by cognitive behavioral elements for 3 weeks). The conclusions have been sustained by Mirza and Deyo [7], who conducted a systematic literature review of four randomized trials comparing lumbar fusion surgery to non-operative care for treatment in CLBP.

Several non-surgical interventions are available for patients with CLBP $[1,2,8,9]$. The guidelines of the National Institute for Health and Clinical Excellence (NICE) [10] recommend cognitive behavioral therapy, exercise therapy, brief educational interventions, and multidisciplinary (bio-psycho-social) treatment. Recently the American Pain Society [11] published an evidencebased clinical practice guideline strongly recommending intensive interdisciplinary rehabilitation with a cognitive behavioral emphasis for patients with non-radicular, low back pain. Although such treatments can be effective, it is not yet known which type of patients benefits most from them $[2,9,12]$.

In The Netherlands, Smeets et al. [9, 13] recently performed a multicenter, randomized clinical trial among 172 patients with non-specific CLBP to determine the effectiveness of three often used approaches in non-surgical treatment. These outpatient treatments were based upon different theoretical models in CLBP: the active physical model, the cognitive behavioral model and a combination of both; they were delivered three times a week during 10 weeks. He then compared these therapeutic modalities to a group of patients on the waiting list. Six-month and 1-year post-treatment results showed that all three active treatments were more effective than no treatment, but no treatment group was more clinically relevant than the other two [9]. The authors concluded that these results could, at least partially, be ascribed to the fact that exposure to the cognitive behavioral treatment components was only $78 \mathrm{~h}$, and not the $100 \mathrm{~h}$ originally recommended in a Cochrane review by Guzman et al. [8]. In the Cochrane review, the authors concluded that intensive multidisciplinary, bio- psycho-social rehabilitation, improves CLBP and physical functioning whereas interventions of less than $100 \mathrm{~h}$ do not yield an additional effect compared to non-multidisciplinary treatment or usual care.

The intensive pain management programme provided by RealHealth NL is based upon cognitive behavioral principles, using a multidisciplinary bio-psycho-social approach, and is of a $100 \mathrm{~h}$ duration. The programme follows the NICE guidelines [10] as well as the American Pain Society recommendations [11]. It is indicated for patients with CLBP for whom surgical or anaesthesiological pain intervention is not an option.

The aim of this article is to evaluate the 1-year followup results of a programme based on the cognitive behavioral approach in patients with CLBP. We conducted a prospective cohort study and compared the results to outcomes of published research on interventions for CLBP.

\section{Materials and methods}

Study design and setting

This is a prospective cohort study with a repeated measures structure to ensure a sufficient degree of internal control. Assessments were made pre-treatment, immediately posttreatment, and to assess whether the effect was sustained, 1 and 12 months after the treatment period. The results of this programme are compared to the outcomes of published research of Fairbank et al. [5] and Smeets et al. [9], on interventions for similar conditions. The pain management programme is part of the Sint Maartenskliniek Nijmegen, but is provided in a hotel facility outside the clinic.

\section{Patients}

Patients entered into the programme consecutively, after a multidisciplinary team of RealHealth (consisting of a psychologist, a physiotherapist and an occupational therapist) screened the patients. These patients had no indication for invasive or anaesthesiological intervention, as confirmed by orthopedic surgeons of the outpatient department of Sint Maartenskliniek, and they were motivated to learn to change their pain behavior.

The patients met the following criteria: low back pain for at least 6 months, no indication for surgical or pain treatment, had no intention of asking for medical treatment for the year following the 2-week programme, age between 20 and 65 years, absence from work for less than 2 years, were motivated to change behavior, were willing to follow the programme and to reside for 2 weeks in the hotel, were able to speak and read Dutch. Exclusion criteria were severe psychiatric disorders and poor physical condition. 
Treatment

The cognitive behavioral programme has been developed by the RealHealth Institute in the United Kingdom and it is a specific pain management programme for patients suffering from CLBP. All sessions in the programme are delivered by the trainers of the multidisciplinary team of RealHealth; the same professionals who did the screening. The full programme comprises an assessment day for intake, the 10-day residential programme with 2 days of follow-up, at 1 and 12 months post-treatment. It is based upon cognitive behavioral principles and involves a $100 \mathrm{~h}$ of participant contact time delivered in a 2-week grouporientated residential setting; around $50 \mathrm{~h}$ of cognitive behavioral training, $35 \mathrm{~h}$ of physical activities, and a $15 \mathrm{~h}$ of education. The main goal of the programme is to increase the patients' ability for self-management and selfefficacy, and with that to address the psychological impact of pain. The perspective is to give people with persistent pain the opportunity to develop techniques and strategies which allows them to minimize the impact of their pain on their daily activities. This is achieved through a combination of a psychological emphasis and physical activities. In general this comprises: a stretch and exercise program, education to define the cause and nature of chronic pain with particular emphasis on the distinction between pain and damage. Patients work on goals to enable them to return to an active lifestyle, using planning and pacing techniques. Cognitive behavioral therapy is given to promote understanding in the link between beliefs, fears, thoughts, and subsequently mood and pain, and to learn techniques to identify unhelpful patterns of thinking and to develop effective responses to challenges. Patients learn to develop a range of relaxation techniques to reduce pain, to aid sleep, and to increase their range and style of strategies to manage increases in pain (http://www.realhealth.org.uk/ what-we-do/pmp).

\section{Outcome assessment: procedure}

Participants provide information about medical history, pain (history, intensity, and duration), medication, general health status, sick leave from work and disability compensation. Standardized and validated self-assessment questionnaires measuring low back pain-related disability, mood, self-management, pain catastrophizing, pain-related fear and quality of life were also filled out as described in the next paragraph. These questionnaires were completed on four occasions: at baseline, (intake within 1 week before start of programme), at the end of 2-week programme, and at 1 and 12 months post-treatment. The participants received no assistance in completing the assessment measures. For intake they were asked to bring the completed questionnaires with them; on the other occasions the participants completed the questionnaires at the treatment location.

\section{Outcome measures}

\section{Roland and Morris Disability Questionnaire (RMDQ)}

The RMDQ [14] is a valid, reliable, and responsive outcome measure for functional disability patients with LBP $[9,15,16]$. This measure is generally used in studies evaluating conservative treatments on patients suffering from CLBP. The total (sum) score ranges between 0 (no disability) and 24 (maximal disability). The minimal clinical important difference (MCID) for clinical relevance is considered to be 5 points [17], although Smeets et al. [9, 13] based their study results on a MCID of 2 points.

\section{Oswestry Disability Index (ODI)}

The ODI [18] is a questionnaire to detect the extent of disability in patients with LBP; it is often used in studies in which orthopedic interventions are evaluated. The widely used measure has been shown to be valid, reliable and responsive in patients with CLBP [15]. It measures the impact of LBP on daily functioning in ten domains. The total (sum) score ranges between 0 and 100, the higher the score the higher the disability. In literature the MCID varied from 4 to 22.1 points when fusion surgery was evaluated [17, 19-22]. The MCID for clinical relevance in this study is considered to be 10 points $[17,20]$.

\section{Pain Self-Efficacy Questionnaire (PSEQ)}

The PSEQ is a 10-item inventory which measures the patient's belief about his/her ability to accomplish a range of activities despite his/her pain [23]. Belief in self-efficacy influences the possibility of effectively using pain-coping strategies. It also measures physical and psychological function and rehabilitation outcome in patients with chronic pain. The measure is used to evaluate the participants' ability to self-manage his/her pain complaints. Scores range from 0 to 60 , with the higher scores indicating stronger self-efficacy beliefs. Validity en reliability has been shown, but no MCID for PSEQ was found in literature. The generally accept MCID of 25\% improvement above mean baseline score has been used for this study.

\section{MOS Short Form-36 Health Survey Questionnaire (SF36)}

The SF36 [24] is a generic instrument to measure the health-related quality of life. The SF36 consists of two component scores: a physical and a mental score, each of which is made up of subscales. The 8 subscales of the SF36 represent generic health concepts, considered to be universal 
and to represent basic human functions and well-being. In this study we only used the SF36 Physical Component Score (SF36 PCS); it is has been used in several studies among patients with chronic pain and has been found valid, reliable and responsive [24]. The total score ranges from 0 to 100 . On the SF36 PCS, a higher score indicates better health. The MCID of the SF36 PCS is reported to be 5.4 [22].

\section{Statistical analysis}

Descriptive statistics were used to present frequencies. A General Linear Model (GLM) repeated measures multivariate analysis of variance was used to identify changes over time using variables measured on at least an interval scale. To show the strength of the within-subject factor Time in the multivariate models of primary outcome measures, $R^{2}$ has been computed. This descriptive statistic indicates the proportion of the variability in the observed data that can be attributed to the treatment. In other words, it is a way to measure treatment effect. Because of the use of questionnaires we anticipated missing data on items. We assumed that these missing data are randomly divided over the different outcome measures. Imputation techniques were used to compensate for these item-missing data. With this, sensitivity analyses are performed to determine the robustness of the outcomes and to estimate the degree and direction of potential confounding. The level of statistical significance was set at 0.05 . All statistical analyses were performed using SPSS, version 12.0.1 for Windows.

Sensitivity analysis Two methods were used for imputation: carrying the last observation forward (CLOF) and imputation of the baseline values for each missing data point (worst-case scenario). We considered the CLOF data the most realistic scenario since one assumes no change over time in the specific item that was substituted and the imputation of baseline values to be the worst-case scenarios, since every effect of the programme was considered to have vanished for that item. We completed the analysis by comparing the patterns of each method with the analysis for those cases with complete data (best-case scenario). When patterns are similar in direction and in the magnitude of estimation of effect size as well, CLOF is considered to be the approach of choice.

Clinical relevance Clinical outcome was defined as mean net change with time in outcome scores as well as the percentage of participants reaching a preset minimal clinical important difference (MCID) for each outcome. The MCID is the threshold for meaningful change; it is defined as the smallest difference that can be considered to be beneficial for the patient and that would result in a change in management by the patient, assuming an absence of excessive side effects or costs [25]. The MCID is often reported as minimal clinical important change (MCIC)
$[17,20]$. Effect sizes were calculated for all outcome measures for the RealHealth programme as well as for the same measures used in published research to indicate the magnitude of treatment effect. It is measured as the standardized difference between the mean post- and pre-treatment score and it takes group variability into account. We used Cohen's $d$ for effect size; it is defined as the difference between means (baseline and follow-up) divided by the pooled standard deviation [26]. An effect size of 1 is equivalent to a change of $1 \mathrm{SD}$ in the sample. The effect sizes are translated into benchmarks for assessing the relative size of change. An effect size $(d)$ of 0.2 is considered small, 0.5 moderate, while 0.8 is a large effect.

To compare results of this cohort study with published research we used Standardized Morbidity Ratios (SMR) to estimate the relative rates of treatment improvement. In this study the SMR is the observed/expected ratio. The expected values have been computed using data from an external reference population [27]. Our reference population came from literature for spinal surgery and rehabilitation programmes for patients treated for CLBP. Formula used to compute SMR is:

\section{$\%$ Observed in RealHealth-NL \\ $\%$ Expected from literature}

The percentages in this formula indicate the number of participants improved. The standard deviation of the difference in the reference data of Fairbank et al. [5] was not reported; therefore, we calculated the variance of the difference using the following formula: $\sigma_{X-Y}^{2}=\sigma_{X}^{2}+\sigma_{Y}^{2}-$ $2 \rho \sigma_{X} \sigma_{Y}$, where $\rho$ is the correlation between the variances of the differences of the variables $X$ and $Y$ in the reference; for this calculation it is assumed to be 0.50 . With the normalized data, a $z$ value is calculated and the percentage patients improved by the intervention can be determined.

\section{Results}

\section{Response}

One hundred fifty-five patients were referred for the RealHealth programme between October 2006 and July 2007: 107 (69\%) were admitted according to the inclusion and exclusion criteria. The 48 excluded patients were referred back to the orthopedic surgeon. Nineteen $(12 \%)$ of the 107 failed to meet the inclusion criteria, 17 (11\%) were included but decided not to join the programme, and 12 $(8 \%)$ were included but wished to postpone their participation until a later time.

In August 2008, all participants had completed the 1-year follow-up. Four of the 107 participants left during the 2-week residential programme: two due to lack of motivation, one 
because of inappropriate behavior during group sessions, and one due to acute illness of the partner. The scores for $77 \%$ $(n=82)$ of all possible items for each participant at each assessment were available for analysis: the best-case scenario. Some data were missing for 21 participants; these missing values were randomly divided among the four assessment moments and the items to be scored. In total 446 of the 5,592 data points were missing $(7.4 \%)$ in the database. For RMDQ 45 data points were missing $(0.7 \%)$ : for ODI, 43 (0.7\%); for PSEQ, $44(0.7 \%)$; and for SF36 PCS, 184 data points $(3.1 \%)$. Imputation techniques were applied to assign a value for the missing data.

Post hoc power analyses, to determine the minimal sample size needed to find a significant effect on the main outcome parameters, showed that a minimum of $26 \mathrm{com}-$ plete data sets was needed to show clinical relevant $(\alpha=0.05, \beta=0.90)$ change on each outcome measure. Since we included the data of all 107 participants for analysis, and compared these results with the best-case scenario (completed cases only), sufficient power was achieved.

\section{Baseline characteristics}

The baseline characteristics for the 107 patients who participated in the RealHealth programme are shown in Table 1 . The mean age was $44( \pm 8.4)$ years; the mean duration of LBP was 12 years, with one 63-year-old patient reporting 52 years of complaints. Females were in the majority (57\%), while $70 \%$ of the participants were currently at work, and one-third had previously undergone surgery for their LBP.

\section{Clinical outcome}

Table 2 shows means and standard deviations (SD) for all outcome measures. Mean outcome scores improved greatly between baseline and directly post-treatment as seen in Table 2. During the following 12 months a continuous, slight improvement in mean scores is seen. Mean improvements between baseline and 12-month follow-up are: 5 points on the RMDQ $( \pm 6.3), 12$ points on the ODI $( \pm 15.2), 10$ points on the PSEQ $( \pm 12.8)$, and 21 points on the SF36 PCS $( \pm 17.6)$.

The $R^{2}$ for the factor time, a measure for treatment effect, for each outcome measure is also presented in Table 2. It is significant for each of the four outcome variables. For both physical functioning scores (ODI and RMDQ), the effects are of similar magnitude as it is for pain self-efficacy (PSEQ). The strongest effect was found for physical functioning related quality of life (SF36 PCS); it was almost one and a half times greater: $R^{2}=0.59$ than the 0.40 found for the other three outcomes.
Table 1 Baseline characteristics reported by participants in RealHealth NL program with chronic low back pain $(n=107)$

\begin{tabular}{ll}
\hline Baseline characteristics & Total $(n=107)$ \\
\hline Sociodemographic & \\
$\quad$ Age, mean (SD, range min-max) in years & $44.1( \pm 8.4,23-60)$ \\
Gender $n(\%)$, male:female & $46(43): 61(57)$ \\
Employment status, $n(\%)$ & \\
Yes:no & $75(70.1): 32(29.9)$ \\
Working: full time & $43(40.2)$ \\
Working: part time & $32(29.9)$ \\
Unemployed because of CLBP & $15(14)$ \\
Unemployed because of other causes & $2(1.9)$ \\
Disability pension & $15(14)$ \\
CLBP history & $12.3( \pm 10.9,1-52)$ \\
Duration of LBP, mean & \\
$\quad$ SD, range min-max) in years & $91(85): 16(15)$ \\
Pain medication $n$ (\%), yes:no & $34(32): 73(68)$ \\
Previous surgery $n(\%)$, yes:no & \\
Baseline values factors related to CLBP & $22.6( \pm 9.2)$ \\
PCS Pain Catastrophizing Scale, mean $(S D)$ & $24.8( \pm 10.4)$ \\
ZSDS Zung Self-Rating Depression Scale, \\
mean (SD)
\end{tabular}

To verify that using the CLOF (carrying the last observation forward) method to compensate for missing data could be applied, the mean scores for each outcome variable were graphically compared as presented in Figs. 1, 2, 3, 4. Each figure presents three scenarios: the best-case scenario $(n=82)$ in which only the patients with no missing values have been included, the worst-case scenario $(n=107)$ in which the baseline value has been inserted for any missing value, and the CLOF method $(n=107)$. In each figure all three curves show the same tendency which supports our assumption that the results are robust, independent of which imputation method was used. They all improve with time, and the curve for the best-case scenario stayed within the confidence intervals of the CLOF curve. Hence, the results obtained using CLOF can be considered as reasonably realistic and can be used to evaluate the RealHealth programme.

The effect sizes for the functional scales, those to assess self-management and quality of life scales, are given in Table 3: these range between 0.8 and 1.3. For our study, all the effect sizes can be classified as large. The clinical relevancy is further supported by the percentage of participants who reached the preset minimal clinical important difference (MCID) for each outcome measure. At least $50 \%$ of the participants showed improvement greater than the reference MCID for all 4 measurements. For a 
Table 2 Mean (SD) for functional disability, self-efficacy and quality of life in CLBP

\begin{tabular}{|c|c|c|c|c|c|}
\hline & Baseline & Last day of 2-week program & 1 month follow-up & 12 months follow-up & $R^{2}$ \\
\hline \multicolumn{6}{|c|}{ Outcome measures } \\
\hline ODI & $41.2(14.5)$ & 35.4 (16.6) & $31.7(16.0)$ & $29.0(17.9)$ & 0.40 \\
\hline RMDQ & $13.9(4.0)$ & $10.1(5.2)$ & $9.2(5.7)$ & $8.9(6.5)$ & 0.39 \\
\hline PSEQ & $33.9(11.5)$ & $42.8(11.0)$ & $42.3(11.2)$ & $43.8(11.3)$ & 0.38 \\
\hline SF36 PCS & $40.2(12.6)$ & $49.6(15.4)$ & $60.9(20.5)$ & $61.3(18.8)$ & 0.59 \\
\hline
\end{tabular}

$R^{2}$ variability attributed to treatment (within-subject factor time), a measure for treatment effect, was analyzed with repeated measures multivariate analyses of variance. CLOF scenario $(n=107)$ was used

$C L O F$ carrying last observation forward, as described in the text

Repeated measures MANOVA are the following: $O D I$ Oswestry Disability Index: $d f(1,106), F=69.20, p<0.001 ; R M D Q$ Roland and Morris Disability Questionnaire: $d f(1,106), F=67.71, p<0.001 ; P S E Q$ pain self-efficacy questionnaire: $d f(1,106), F=65.14, p<0.001 ; S F 36$ PCS MOS Short Form 36 Physical Component Scale: $d f(1,106), F=152.08, p<0.001$

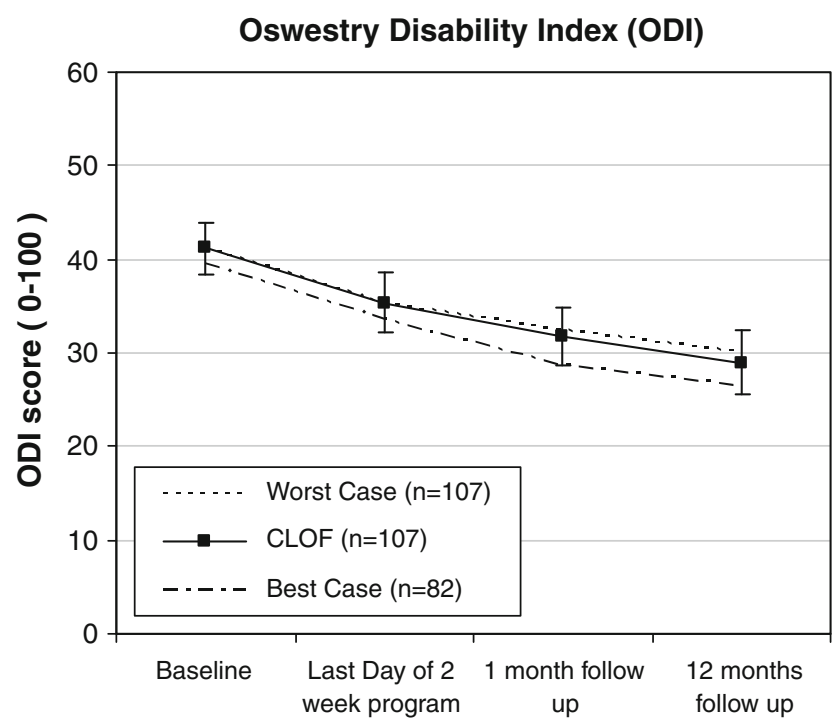

Time

Fig. 1 Oswestry Disability Index $(O D I)$. Graphic trends of three scenarios: the worst case (imputation of baseline value, $n=107$ ), $C L O F$ (carrying the last observation forward, $n=107$ ), and the bestcase scenario (completed cases only, $n=82$ )

reduction of 5 points on RMDQ [17], 49\% showed a relevant improvement in daily functioning 12 months after the treatment. A clinical relevant improvement of 10 points or more on ODI $[17,20]$ was seen in $56 \%$ of the participants. The MCID for self-management of pain (PSEQ) was set a $25 \%$ (8.5 points) of the mean baseline score; 57 participants (53\%) showed a relevant improvement. For the quality of life related to physical functioning (SF36 PCS) 86 participants $(80 \%)$ showed a meaningful improvement of 5.4 points [22] or more.

In order to be able to compare the results of the RealHealth programme with those found in the literature, both the effect sizes and standardized morbidity ratios (SMR), based on the data provided in the article, needed to be

\section{Roland \& Morris Disability Questionnaire (RMDQ)}

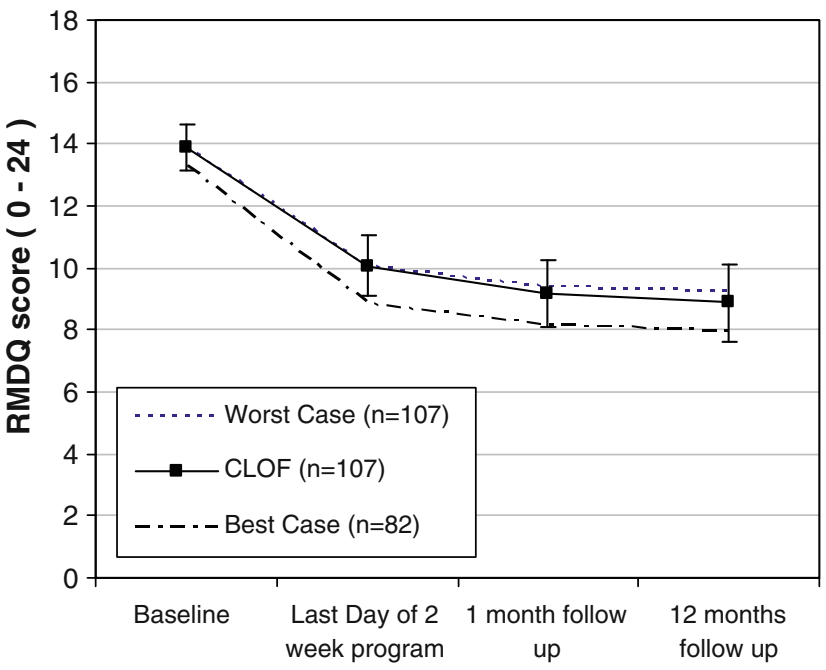

Time

Fig. 2 Roland and Morris Disability Questionnaire (RMDQ)

calculated (Table 3). No one study used all of our outcome variables. For the cognitive behavioral treatment group reported by Smeets et al. [9] the effect size is small $(d=0.2)$. To compare with the results of the UK MRC spine stabilization trial [5], we calculated the Cohen's $d$ for the fusion group and the rehabilitation group separately. As can be seen in Table 3, the effect sizes for both interventions for the ODI are moderate (surgery $d=0.7$ and rehabilitation $d=0.5$ ) as is that for the SF36 PCS (rehabilitation $d=0.6$ ). Only the Cohen's $d$ for the SF36 PCS for the group which had had a surgical intervention is considered large $(d=0.8)$.

For the SMR: first the literature studies the percentage of patients who improved was determined for the literature studies and for the RealHealth study. Subsequently, the SMR was calculated, as shown in Table 3. To compare with the cognitive behavioral treatment of Smeets et al. [9] 


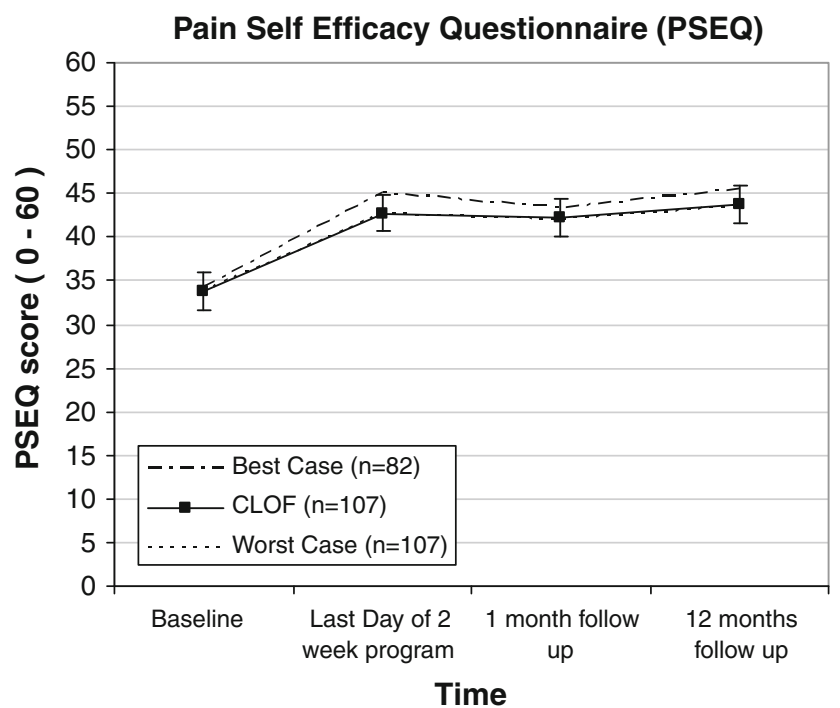

Fig. 3 Pain Self-Efficacy Questionnaire (PSEQ)

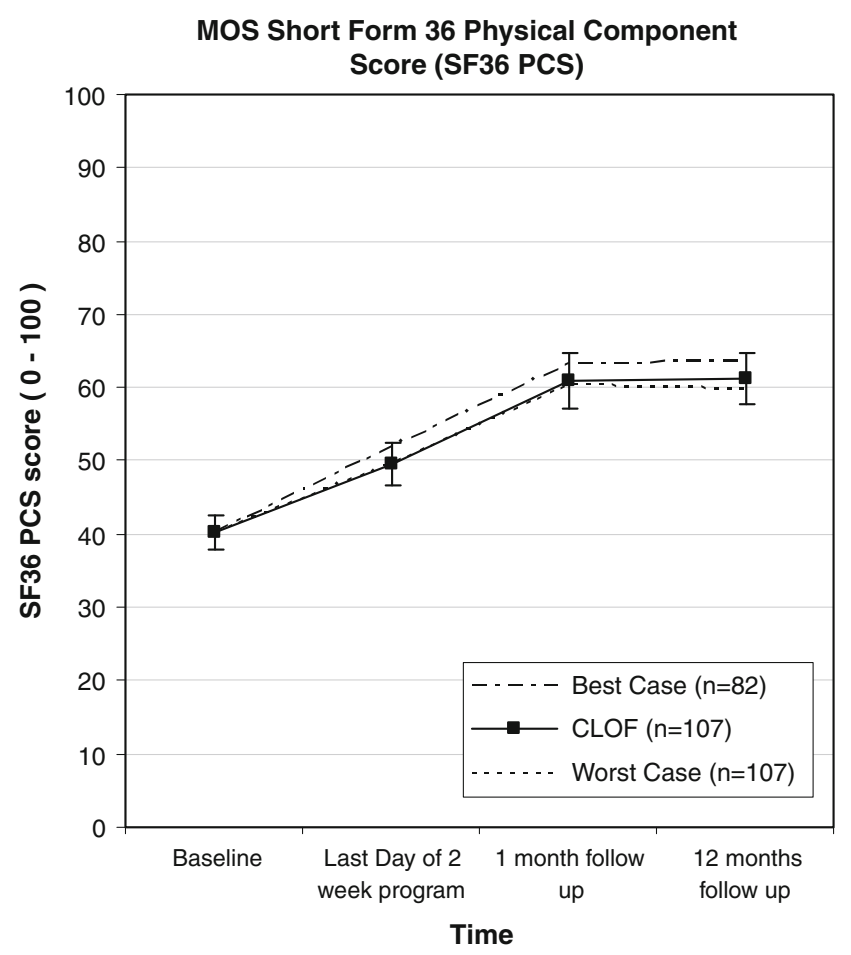

Fig. 4 SF36 Physical Component Score (SF36 PCS)

a 2-point MCID was used, as reported in that study. The calculated SMR of $136 \%$ indicates that $36 \%$ more of the participants of the RealHealth programme showed improvement on the RMDQ. In comparison to the rehabilitation treatment of Fairbank et al. [5], the SMR of 107\% indicates that a slightly larger percentage of the RealHealth participants showed improvement on the ODI. The SMR of 98\% for the comparison between surgical treatment [5] and the RealHealth programme indicate that a slightly larger percentage of the surgical intervention group showed improvement on the ODI. More participants of the RealHealth programme $(10 \%$ when compared with surgical intervention group; $18 \%$ for the rehabilitation group) showed improvement on the SF36 PCS. No comparative literature study could be found for the PSEQ.

\section{Discussion}

The aim of this article was to evaluate the 1-year follow-up results in patients with CLBP after a cognitive behavior based programme provided by RealHealth-Netherlands. This programme follows the international guidelines $[10,11]$, and is indicated for patients for whom surgery or anaesthesiology is not an option. These results were compared to the outcomes for interventions for patients with CLBP found in published research. More than half of the prospective cohort of RealHealth participants showed improvement in daily functioning, learned to manage their chronic disabling low back pain, and for a tremendous amount of the cohort $(80 \%)$ their quality of life improved significantly. All these clinically relevant results were sustained at 1-year follow-up, and were meaningful to patients. These improvements were greater than the reference MCID for all outcome measures. The effect of the treatment is further established by the large effect sizes (Cohen's $d$ ranging from 0.8 to 1.3) found for the four outcome measures, which indicate a treatment effect and by the SMRs used to compare the RealHealth results to two other studies in which patients were treated for CLBP; these ranged from 98 to $136 \%$. When compared to other multidisciplinary rehabilitation programmes, the results for daily functioning favor the RealHealth programme, and were comparable to those achieved after fusion surgery.

Comparison with related research: multidisciplinary rehabilitation programmes

We compared the results of the RealHealth programme to the interventions recently conducted in The Netherlands by Smeets et al. [9], in which the patients were randomized among three treatment groups (the active physical, the cognitive behavioral and a combination of both) was also compared to a group of patients on the waiting list. Oneyear post-treatment results showed that all three active treatments were more effective than no treatment; however, no treatment group had greater clinical relevant improvement than the other two. The cognitive behavioral arm is most similar to the RealHealth programme; therefore, those results were compared to those obtained in the present study. The baseline level of functional disability, 
Table 3 Clinical relevancy after 12 months

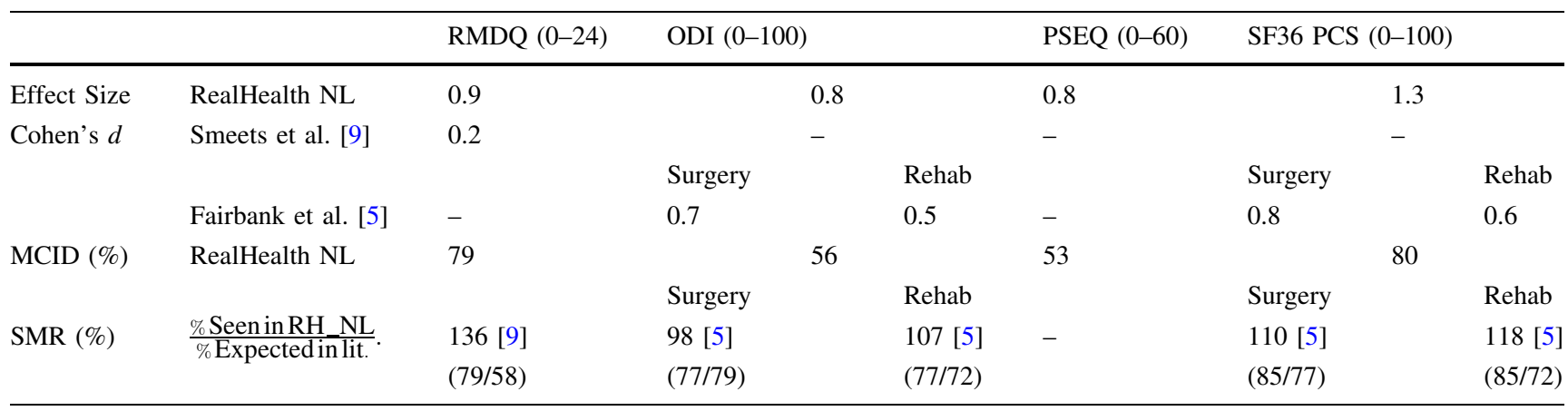

Calculated percentages of the RealHealth program participants who reached the MCID. Effect sizes and SMR as relative rate for treatment improvement were calculated for the RealHealth programme as well as for the external reference populations found in literature [5, 9]

MCID minimal clinical important difference: MCID for RMDQ $=2$ points [9], MCID for ODI $=10$ points [17, 20], MCID for PSEQ $=8.5$ (based on $25 \%$ of the mean baseline score), MCID for SF36 PCS $=5.4$ point [22]; SMR standardized morbibidity ratio

measured by the RMDQ, is comparable to that of our study population (both $14 \pm 4$ ). Depending on the therapy given, the Smeets et al. study reported at 1-year follow-up a mean improvement of 2-4 points on the RMDQ; this is lower than the 5 points improvement reported in the RealHealth study. When a 2-point reduction on RMDQ was used to show clinical relevancy, $58 \%$ of those patients reached this threshold 1-year post-treatment. When we applied the same criterion, $79 \%$ of our patients showed a clinically relevant improvement.

An extremely small effect size was calculated for the cognitive behavioral group of Smeets et al. for functional disability ( $d=0.2)$ versus the large effect size $(d=0.9)$ of the programme of RealHealth, which is favorably sustained by the SMR of $136 \%$. The small effect size found is possibly due to the heterogeneous patients, as shown in the relatively large standard deviation of 4 points. The authors of that study also mentioned the limited exposure to cognitive treatment $(78 \mathrm{~h})$. The results of the RealHealth programmes seems to validate the guideline value of $100 \mathrm{~h}$ exposure to cognitive behavioral techniques [8].

Comparison with related research: fusion surgery

We also compared the results of the RealHealth programme to those reported in the MRC spine stabilization trial by Fairbank et al.[5]. They randomized patients to either a surgical stabilization (spinal fusion) or an intensive rehabilitation. Those authors concluded there is no clear evidence that primary spinal fusion surgery was any more beneficial than intensive rehabilitation. We chose that study to determine whether an intensive cognitive behavioral pain management programme could be an alternative for patients with longstanding CLBP. In studies in which evaluations of surgical interventions, such as spinal fusion, were reported the primary disability outcome measure is usually is the ODI. When comparing the baseline characteristics for disability, we found that the surgical group had a score of 47 , whereas the mean score in the rehabilitation group was 45 , which are reasonably comparable to the 41 points found in the current study. Although in the present study an orthopedic surgeon confirmed that there was no indication for an invasive treatment, this baseline characteristic implies that the RealHealth programme treated patients with equivalent severity of disability.

At 2-year follow-up a 13-point improvement in ODI score was found for the surgical group and 9 points for the rehabilitation group. Although we had only 1-year results, the current study shows 12 points improvement in ODI, which compares favorably with both the rehabilitation and surgical intervention groups of the UK MRC trial. Considering the generally accepted MCID, a 10-point improvement is needed to show meaningful clinical relevance $[17,20]$.

In short, the RealHealth programme seems to be superior to the surgical or rehabilitation treatments: the effect size for quality of life related to physical functioning is extremely large $(d=1.3)$ and considered large for disability $(d=0.8)$. The comparison for reduction of disability is equivalent: 0.8 vs. 0.7 and 0.6 , for surgical and rehabilitation treatment, respectively. This conclusion is substantiated since $80 \%$ of the RealHealth participants reported a meaningful improvement in quality of life after a year and $56 \%$ reported that they functioned better than previously. Therefore, the RealHealth programme seems to be an important alternative intervention when fusion surgery is considered. The improvement found with the RealHealth programme in comparison to the intensive outpatient rehabilitation programme reported by Fairbank et al. [5] may be explained by the fact that only $75 \mathrm{~h}$ of outpatient rehabilitation had been offered during a 3-week period. Once more the RealHealth programme's $100 \mathrm{~h}$ 
seems to validate the guideline value of $100 \mathrm{~h}$ exposure to cognitive behavioral techniques [8].

\section{Limitations of the study}

This study also has its limitations. According to the inclusion criteria used for the cognitive behavioral RealHealth programme, patients are required to have no indication for surgical treatment and had to be motivated to change their behavior with regard to their pain complaints. This implies that a selection bias might have been introduced, especially since the Sint Maartenskliniek is a specialized hospital to which patients are often referred for second opinion or as a last resort. This limits the generalizability of the results. However, this type of treatment will not be efficacious if patients are not motivated to change their behavior. Therefore, it is possible that in general practice, the results could be less favorable since the inclusion criteria would not be as narrowly defined.

In the study reported here, several precautions were taken to enhance internal validity and minimize the potential influence of bias and confounding on the outcome factors these include multivariable adjustment, sensitivity analysis and standardization. Emphasis should to be placed on the use of sensitivity analyses, which was motivated by the fact that there were item-missing data on the outcome measurements. Although no systematic pattern was discerned, these missing data need to be taken into consideration when the results of the study are estimated and interpreted. The analyses indicate the ranges within which the effects may be expected and do not give an exact estimate of the magnitude of the effect. The use of SMR provides information about the magnitude of effect compared to other studies and/or populations. An SMR, a rate ratio is, thus an estimate of relative treatment improvement. However, since an external reference has been used to calculate the SMRs, discrepancies in the population characteristics are likely and it is not possible to judge whether sufficient controls have been conducted to enhance the internal validity within the studies reported in the literature. However, the SMRs can be accepted as a technique to enable a measure of comparison.

In summary, the comparisons with other treatments seem to indicate that the intensity, duration and frequency of a cognitive behavioral pain management programme may be the most important key to success. The results of the short an intensive RealHealth programme are promising, but further research is needed to determine which factors of CLBP are influenced by this treatment programme, and to answer the frequently reported question [2, 9, 12]: 'Which type of patient benefits the most?' It is interesting to note that even participants with longstanding CLBP complaints (mean complaint duration in the
RealHealth programme was 12 years) seem to experience benefits from this short, intensive programme, suggesting that CLBP duration is not an important factor for the management of CLBP. Factors such as mood, fear of movement, catastrophizing, coping strategies and selfmanagement, which will be analyzed in a following study, do seem to be important factors. Although the comparison technique used in this study has some drawbacks, it is justified to conclude that the RealHealth programme seems to allow CLBP patients to achieve clinically relevant improvement; the treatment effect seems to be comparable to spinal surgery and to achieve better results than less intensive rehabilitation programmes.

Acknowledgments The authors thank the multidisciplinary treatment team at Realhealth NL who were responsible for the intake procedure and training the participants in pain management programme. Particular thanks are for Cissy Matthijssen (RealHealth NL), Lauren White (RealHealth UK) for their assistance in completing the database, and Patricia G. Anderson for her editorial assistance.

Open Access This article is distributed under the terms of the Creative Commons Attribution Noncommercial License which permits any noncommercial use, distribution, and reproduction in any medium, provided the original author(s) and source are credited.

\section{References}

1. van Tulder MW, Koes BW, Bouter LM (1995) A cost-of-illness study of back pain in The Netherlands. Pain 62:233-240

2. van Tulder MW, Ostelo R, Vlaeyen JW et al (2000) Behavioral treatment for chronic low back pain: a systematic review within the framework of the Cochrane Back Review Group. Spine 25:2688-2699

3. Smeets RJ, Wade D, Hidding A et al (2006) The association of physical deconditioning and chronic low back pain: a hypothesisoriented systematic review. Disabil Rehabil 28:673-693

4. Picavet HS, Schouten JS (2003) Musculoskeletal pain in the Netherlands: prevalences, consequences and risk groups, the DMC(3)-study. Pain 102:167-178

5. Fairbank J, Frost H, Wilson-MacDonald J et al (2005) Randomised controlled trial to compare surgical stabilisation of the lumbar spine with an intensive rehabilitation programme for patients with chronic low back pain: the MRC spine stabilisation trial. BMJ 330:1233

6. Koes BW (2005) Surgery versus intensive rehabilitation programmes for chronic low back pain. BMJ 330:1220-1221

7. Mirza SK, Deyo RA (2007) Systematic review of randomized trials comparing lumbar fusion surgery to nonoperative care for treatment of chronic back pain. Spine (Phila Pa 1976.) 32:816823

8. Guzman J, Esmail R, Karjalainen K et al (2002) Multidisciplinary bio-psycho-social rehabilitation for chronic low back pain. Cochrane Database Syst Rev CD000963

9. Smeets RJ, Vlaeyen JW, Hidding A et al (2008) Chronic low back pain: physical training, graded activity with problem solving training, or both? The one-year post-treatment results of a randomized controlled trial. Pain 134:263-276

10. National Institute for Health and Clinical Excellence (2009) Low back pain: early management of persistent non-specific low back pain. National Collaborating Centre for Primary Care, London 
11. Chou R, Loeser JD, Owens DK et al (2009) Interventional therapies, surgery, and interdisciplinary rehabilitation for low back pain: an evidence-based clinical practice guideline from the American Pain Society. Spine (Phila Pa 1976.) 34:1066-1077

12. McCracken LM, Turk DC (2002) Behavioral and cognitivebehavioral treatment for chronic pain: outcome, predictors of outcome, and treatment process. Spine 27:2564-2573

13. Smeets RJ, Vlaeyen JW, Hidding A et al (2006) Active rehabilitation for chronic low back pain: cognitive-behavioral, physical, or both? First direct post-treatment results from a randomized controlled trial. BMC Musculoskelet Disord 7:5

14. Roland M, Morris R (1983) A study of the natural history of back pain. Part I: development of a reliable and sensitive measure of disability in low-back pain. Spine 8:141-144

15. Roland M, Fairbank J (2000) The Roland-Morris Disability Questionnaire and the Oswestry Disability Questionnaire. Spine 25:3115-3124

16. Ostelo RW, de Vet HC, Knol DL et al (2004) 24-Item RolandMorris Disability Questionnaire was preferred out of six functional status questionnaires for post-lumbar disc surgery. J Clin Epidemiol 57:268-276

17. Ostelo RW, Deyo RA, Stratford P et al (2008) Interpreting change scores for pain and functional status in low back pain: towards international consensus regarding minimal important change. Spine 33:90-94

18. Fairbank JC, Couper J, Davies JB et al (1980) The Oswestry low back pain disability questionnaire. Physiotherapy 66:271273
19. Wilson-MacDonald J, Fairbank J, Frost H et al (2008) The MRC spine stabilization trial: surgical methods, outcomes, costs, and complications of surgical stabilization. Spine 33:2334-2340

20. Ostelo RW, de Vet HC (2005) Clinically important outcomes in low back pain. Best Pract Res Clin Rheumatol 19:593-607

21. Tafazal SI, Sell PJ (2006) Outcome scores in spinal surgery quantified: excellent, good, fair and poor in terms of patientcompleted tools. Eur Spine J 15:1653-1660

22. Glassman SD, Gornet MF, Branch C et al (2006) MOS Short Form 36 and Oswestry Disability Index outcomes in lumbar fusion: a multicenter experience. Spine J 6:21-26

23. Nicholas MK (2007) The pain self-efficacy questionnaire: taking pain into account. Eur J Pain 11:153-163

24. Ware JE Jr, Sherbourne CD (1992) The MOS 36-item short-form health survey (SF-36). I. Conceptual framework and item selection. Med Care 30:473-483

25. Redelmeier DA, Guyatt GH, Goldstein RS (1996) Assessing the minimal important difference in symptoms: a comparison of two techniques. J Clin Epidemiol 49:1215-1219

26. Cohen J (1988) Statistical power analysis for the behavioral sciences, 2nd edn. Lawrence Earlbaum Associates, Hillsdale, NJ

27. Rothman KJ, Greenland S, Lash TL (2008) Modern epidemiology, 3rd edn. Lippincott Williams and Wilkins, Philadelphia, pp 664681 